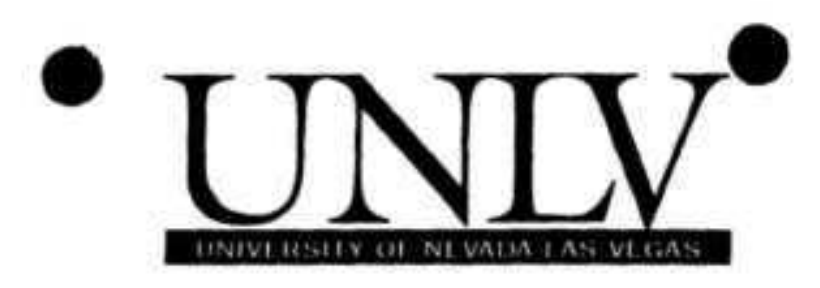

Melanie Becker

Office of Basic Energy Sciences

Division of Materials Sciences and Engineering (SC-13)

U.S. Department of Energy; 19901 Germantown Road; Germantown, MD 20874-1290

Ms. Becker:

Under this cover you will find a copy of my final report summarizing the results from the past four years of an EPSCoR State-DOE Laboratory Partnership Award Studies of CorrelatedElectron Systems in High Magnetic Fields and at High Pressures. This is DOE Award Number DE-FG02-00ER45835 with a total award amount of $\$ 219,033$ for the period $8 / 00-8 / 03$ with a one year no cost extension). As this is the end of the award, I would like to thank DOE-BES and the EPSCoR program for the opportunity to perform the research outlined in this report. I feel overall that the project has been a great success and I look forward to continuing a working relationship with the Office of Basic Energy Sciences.

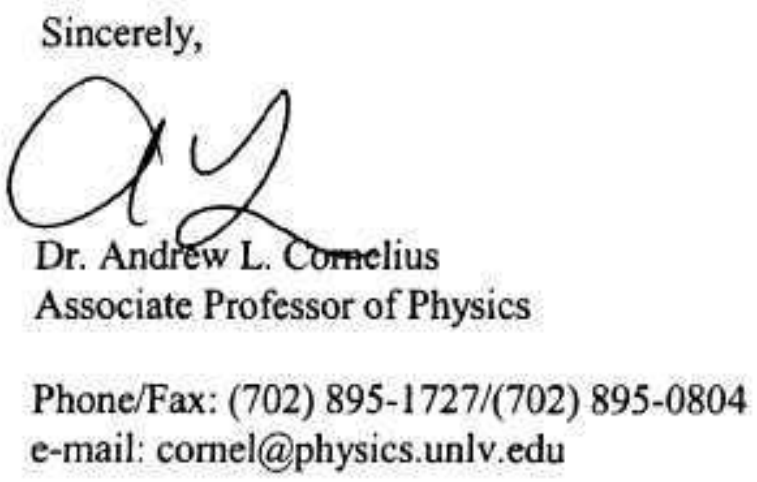




\section{Studies of Correlated-Electron Systems in High Magnetic Fields and at High Pressures}

The following summarizes the results from the past four years of an EPSCoR State-DOE Laboratory Partnership Award Studies of Comelated-Electron Systems in High Magnetic Fields and at High Pressures (DOE Award Number DE-FG02-00ER45835 with a total award amount of $\$ 219,033$ for the period $8 / 00-8 / 03$ with a one year no cost extension). Numerous publications and presentations came from the funding, and the PI received recognition in the form of an invited presentation at the 2003 DOE/EPSCoR conference EPSCOR Powered: Pathways to Success in Albuquerque, New Mexico.

\section{A. Observation of Multiple Energy Scales in $\mathbf{Y b A l}_{\mathbf{3}}$}

$\mathrm{YbAl}_{3}$ is an intermediate valent (IV) compound with a Kondo temperature $T_{K}$ in excess of 500 $\mathrm{K}$ and a rather low conduction electron density of $n_{c}{ }^{-0.5}$ /atom. ${ }^{1}$ Recent theoretical studies ${ }^{2-4}$ of the Kondo Lattice Model (KLM) and Anderson Lattice Model (ALM) suggest that the thermodynamic properties can differ in at least two ways from the predictions of the Anderson Impurity Model (AIM). First, a new low temperature scale $T_{\text {con }}$ for the onset of Fermi liquid coherence with $T_{\text {con }} \ll$ $T_{K}$. Second, as $n_{c}$ decreases, the crossover from low temperature Fermi liquid behavior to bigh temperature local moment behavior becomes slower than predicted for the AIM. Though all of the theories predict $T_{\text {coh }} \propto T_{K}$, there is no universal relation between the two. We have experimentally observed both effects in $\mathrm{YbAJ}_{3} .{ }^{1}$ First, the crosscver from low temperature Fermi liquid behovior to high temperature local moment behavior is slower (i.e.,more gradual) than predicted for the AIM. Second, snomalies (relative to the AM) in the magnetization, susceptibility specific heat, and magnetotransport occur below $30-40 \mathrm{~K}$, which is the temperature scale $T_{\text {con }}$ for the onset of coherent Fermi liquid $T^{2}$ behavior in the resistivity. For $T$ \& $T_{\text {enh }}$ we indeed find that the magnetization "crosses" over from the zero feld energy scale $\left(T_{c o h}\right)$ to the high temperature energy scale $T_{K}$ at a magnetic field $B^{*} \approx 40 \mathrm{~T}\left(\approx k_{B} T_{\text {coh }} / \mu_{B}\right)$ with ittle change in the shape of the Fermi surface (however the effective masses are all reduced by a factor of 2-3 relative to their low field value ${ }^{5}$ ). This is the first direct observation of the crossover between the $T_{c o h}$ and $T_{K}$ energy scales in an IV compound.

In Kondo systems, the magnetic susceptibility $\chi(T)$ displays universal behavior a function of $T / T_{K}$, and from the maximum in $\chi(T)$, one can obtain a value for $T_{K}$. In Fig. 1 results on $\mathrm{YbAJ}_{3}$ are shown. At $250 \mathrm{~K}\left(T>T_{\text {coh }}\right)$, linearity is observed up to the highest field of around 60 $T$. At $4 \mathrm{~K}\left(T<T_{c \infty h}\right)$ there is deviation from linearity around $B^{*} \approx 40 \mathrm{~T}$ as can be clearly in the difference plot shown in the inset to Fig. 1. The low field $(B=0.1 \mathrm{~T})$ magnetic susceptibility measured in a SQUID magnetometer show maxima at $T=15 \mathrm{~K}$ and $125 \mathrm{~K}$ that arb related to $T_{c o h}$ and $T_{K}$ respectively. From the slope of the $M$ versus $B$ curves at low fields, good agreement is seen with the SQUID dste. Both of these date sets shows good apreement to an NCA calculation using $T_{K}=565 \mathrm{~K}$ for $T>75 \mathrm{~K}$ but deviate at lower temperatures. This is due to the new lower energy scale $T_{\text {con }}$ scale at low temperatures. For high felds, however, the slope of the $M$ versus $B$ for $B^{*}>40 \mathrm{~T}$ ( $\approx k_{B} T_{\operatorname{coh}} / \mu_{B}$ ) curveg show good agreement to the calculations. This is clear evidence of a sudden change from the large energy scale to the small energy scale in bigh fields at low temperatures. These results show that magnetic susceptibility can be used to determine BOTH $T_{K}$ and $T_{\text {coh }}$. 

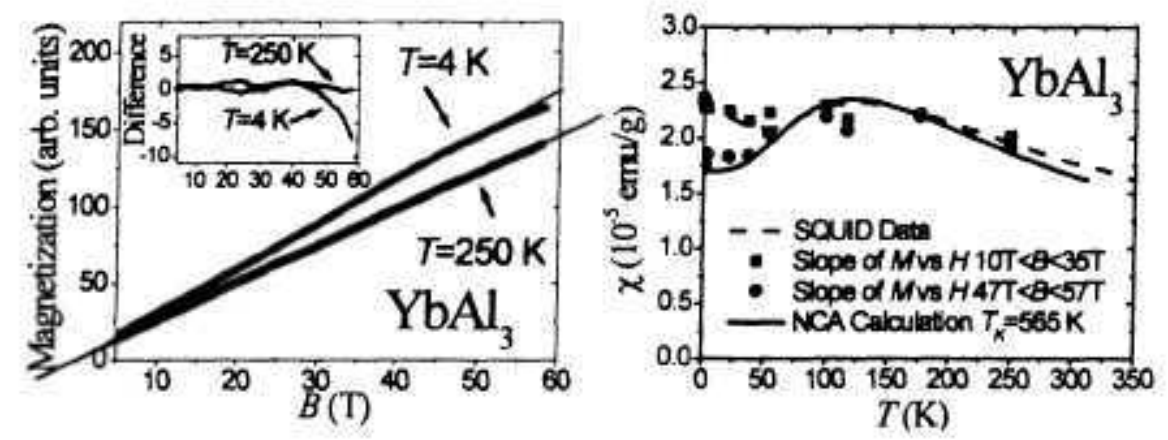

Figure 1: (left) The magnetization as a function of field for two different temperatures. The lines are linear fits to the data in the range $(10 \mathrm{~T}<B<35 \mathrm{~T})$. The inset show the difference of the raw data and the linear fit. (right) Plots of the magnetic susceptibility $\chi(T)$ for very low fields (SQUID data), low fields $(10 \mathrm{~T}<B<35 \mathrm{~T}$ ), high fields (10 $\mathrm{T}<B<35 \mathrm{~T}$ ) and the results of an NCA calculation with $T_{K}=565 \mathrm{~K}$.

\section{B. Magnetically Mediated Superconductivity in CeRhIn}

One of the more extensively studied heavy fermion systems in the past few years is CeRhIn and related compounds. CeRhIn 5 crystallizes in the quasi-two-dimensional (quasi-2D) tetragonal HoCoGa $a_{5}$-type structure and displays antiferromagnetic $(\mathrm{AF})$ ordering at $T_{N} \approx 3.8 \mathrm{~K}$. The electronic specific heat coefficient $\left(\gamma \geq 420 \mathrm{~mJ} / \mathrm{mol} \mathrm{K}^{2}\right)$ determined for $T>T_{N}$ makes CeRhIn $\mathrm{n}_{5}$ a moderately heavy-fermion compound. The application of pressure induces a superconducting transition at $T_{c}=2.1 \mathrm{~K}^{6}$. The order of magnitude larger $T_{c}$ compared to cubic $\mathrm{CeIn}_{3}$ is attributed to the quasi-2D crystal structure. The PI has been submitted the second manuscript on CeRhIn ${ }_{5}{ }^{7}$ and he has been actively studying it and related compounds as outlined below. The crystal structure of $\mathrm{CeRhIn}$ can be viewed as $\left(\mathrm{CeIn}_{3}\right)\left(\mathrm{RhIn}_{2}\right)$ with alternating layers of cubic $\left(\mathrm{CeIn}_{3}\right)$ and tetragonal $\left(\mathrm{RhIn}_{2}\right)$ stacked along the $c$-axis. As theoretical models suggest that the SC state is enhanced for $2 \mathrm{D}$ magnetic correlations relative to $3 \mathrm{D}$ correlations, ${ }^{8}$ the larger $T_{c}$ in CeRhIn ${ }_{5}$ relative to cubic $\mathrm{CeIn}_{3}$ might be due to the difference in crystal structures. The magnetic and electronic properties of CeRhIn , as determined by heat capacity and de Haas-van Alphen measurements, can be well explained by postulating the formation of an anisotropic spin density wave (SDW) consistent with neutron measurements, ${ }^{9}$ that opens a gap in the Fermi surface at low temperatures. ${ }^{7}$ The anisotropy leads to the speculated quasi-2D nature of the magnetic interactions is a result of the tetragonal crystal structure of CeRhIng.

We have studied the elastic properties of $\mathrm{Ce}_{n} M \operatorname{In}_{2 n+3}(M=\mathrm{Rh}$, Ir and Co) with $n=1$ or 2 under hydrostatic and quasihydrostatic pressures up to $15 \mathrm{GPa}$ using $\mathrm{x}$-ray diffraction. The data collected on $\mathrm{CeRhIn}_{5}$ is shown in Fig. 2. Since the maximum volume compression is only of the order of $10 \%$, the $V(P)$ data has been fit using a least squares fitting procedure to the first order Murnaghan equation of state. For the room temperature $(T=295 \mathrm{~K})$ data in Fig. 2, we find $B_{0}=78.4 \pm 2.0 \mathrm{GPa}$ and $B_{0}^{\prime}=5.6 \pm 0.6$. The addition of $M \mathrm{In}_{2}$ layers to the parent CeIn 3 compound is found to stiffen the lattice since $B_{0}=67 \mathrm{GPa}$ for $\mathrm{CeIn}_{3} .{ }^{10}$ The $c / a$ data displays a double maxima as a function of pressure. An intriguing explanation for this result is that there are two superconducting states in CeRhIns: a higher temperature quasi-2D state at low 
pressure and a lower teraperature more $3 D$ state at higher pressures. This acenario is supported by the experimental finding a two waxima in $T_{c}(P)$ in CeFlins and Is substituted samples; ${ }^{11,12}$ the maxima in $T_{c}(P)$ for CeRhins occur at nearly the same pressures as the rataxima in $\mathrm{c} / \mathrm{a}$. As room temperature lattice properties are being correlated to superconductivity at low temperatures, it is important to make sure that the same lattice properties messured at room temperature hold at low temperature. A measurement at $10 \mathrm{~K}$ shown in Fig. 2 also displays the dotible maxima in $\mathrm{c} / \mathrm{a}$ validating the use of room temperature values.

By plotting the maximum values of the superconducting transition temperatute $T_{c}$ versus $c / a$, we are able to expand upon the proposed linear relationship between the quantities by Pagliuso et a ${ }^{14}$ In Fig. 2, I plot normalized values of $T_{c}$ versus $\Delta(c / a)$ in Fig. 2 , where $T_{c}$ is normalized by $T^{*}$ and $\Delta(c / a)$ is found by subtracting a value $(c / a)^{*} . T^{*}$ was chosen as $2 \mathrm{~K}$ for Ce $M \mathrm{I}_{\mathrm{r}}$ and $\mathrm{Ce}_{2} M \mathrm{In}_{\mathrm{B}}$ as it is approximately $T_{o f}$ for $\mathrm{CeColn}_{5}{ }^{15}$ and we don't expect much variation in $T_{K}$ for these compounds. $T^{*}=20 \mathrm{~K}$ was used for PuMGas as we expect an order of magnitude increase in $T_{K}$ for Pu compounds relative to Ce compounds (the PuMGas data is from Wastin et ad. ${ }^{16,17}$ ). (c/a)" was chosen in such a way to shift the curves on top of each other. The universality is readily apparent. The ambient pressure "misplacement" of $\mathrm{Ce}_{2} \mathrm{RhI}_{8}$ (AF order at ambient pressure) now can be explained by the pressure induced superconductivity and the uriversal line now goes through the high preseure $\mathrm{Ce}_{2} M \mathrm{In}_{6}$ data. While $\mathrm{Ce}_{2} \mathrm{IrIn}_{\mathrm{I}}$ does not display superconductivity, the value of $c / \alpha$ reaches a nearly constant value above $5 \mathrm{GPa}$ and we have plotted a point assuming $T_{c} \neq 0$ at high pressure. This assumption gains validity as these results would predict that superconductivity

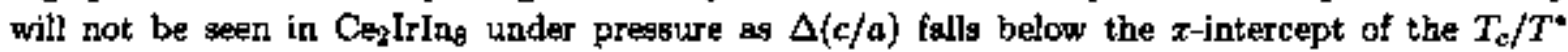
versus $\Delta(c / a)$ line. These results explain the lack of superconductivity in CengIng and predict that $T_{\varepsilon}$ should increase dramatically in $\mathrm{Ce}_{2} \mathrm{CoIn}_{8}$ at high pressure. Comparing the results to Pu-based superconductors shows a universal $T_{c}$ yersus $c / a$ behavior when these quantities are normalized by sppropriate quantities consistent with what is expected of magnetically mediated superconductivity.

The use of high presure, high magnetic fields, and chemical substitution to alter the electronelectron (bybridization) interactions can suppress long range magnetic order that terminatea at a quantum critical point (QCP). Near the QCP, a variety of exotic behavior can be observed including: short range magnetic order, non-Fermi liquid (NFL) behavior, heavy fermion behavior and superconductivity. We compare our beat capacity data using chemical substitution to both theoretical calculations of the anisotropic $2 \mathrm{D}$ Ising model and the high pressure data of Fischer et al. where superconductivity is observed. ${ }^{13}$ To discuss the data in terms of short range magnetjc interactions, we will look at the calculations for a 2D Jaing model on a square lattice with two magnetic intersction energies $E_{\text {in }}$ and $E_{\text {out }}$ that correspond to the magnetic interaction energies along the two orthogonal directions. ${ }^{19}$ To a first approximation, it seerns reasonsbie to qualitatively deacribe our data using $E_{\text {in }}$ as the interaction in the basal plane and $E_{\text {out }}$ as the out of plane (interlayer) interaction. We fix $E_{\text {in }} / 2=T_{N}$ and vary $E_{\text {ord }}$ with the results shown in Fig. 2 where a dramatic effect on $C_{m} / T$ is seen as $T_{N}$ moves to lower temperatures and a Schottky-like maximum (or hump) becomes clearly visible. In the calculatione, the heat capacity for the case $E_{\text {in }} / E_{\text {out }}=\infty$ shows no long range order and is identical to that of a two-state Schottky anomaly with an energy difference of $E_{\text {in }}$ between the two levels. The evolution of the $2 D$ Ising calculation is in excellent agreement with the data taken on $\mathrm{Ce}_{1-x} \mathrm{La}_{x} \mathrm{Fh}_{n_{5}}$ shown in Fig. 2. In this scenario, as Lo is doped for $\mathrm{Ce}$, short range in plane magnetic correlations remain while thoae along the $c$-axis are weakened considerably by the disorder. The long range order is suppressed (disappears around $x=0.40$ ) by 

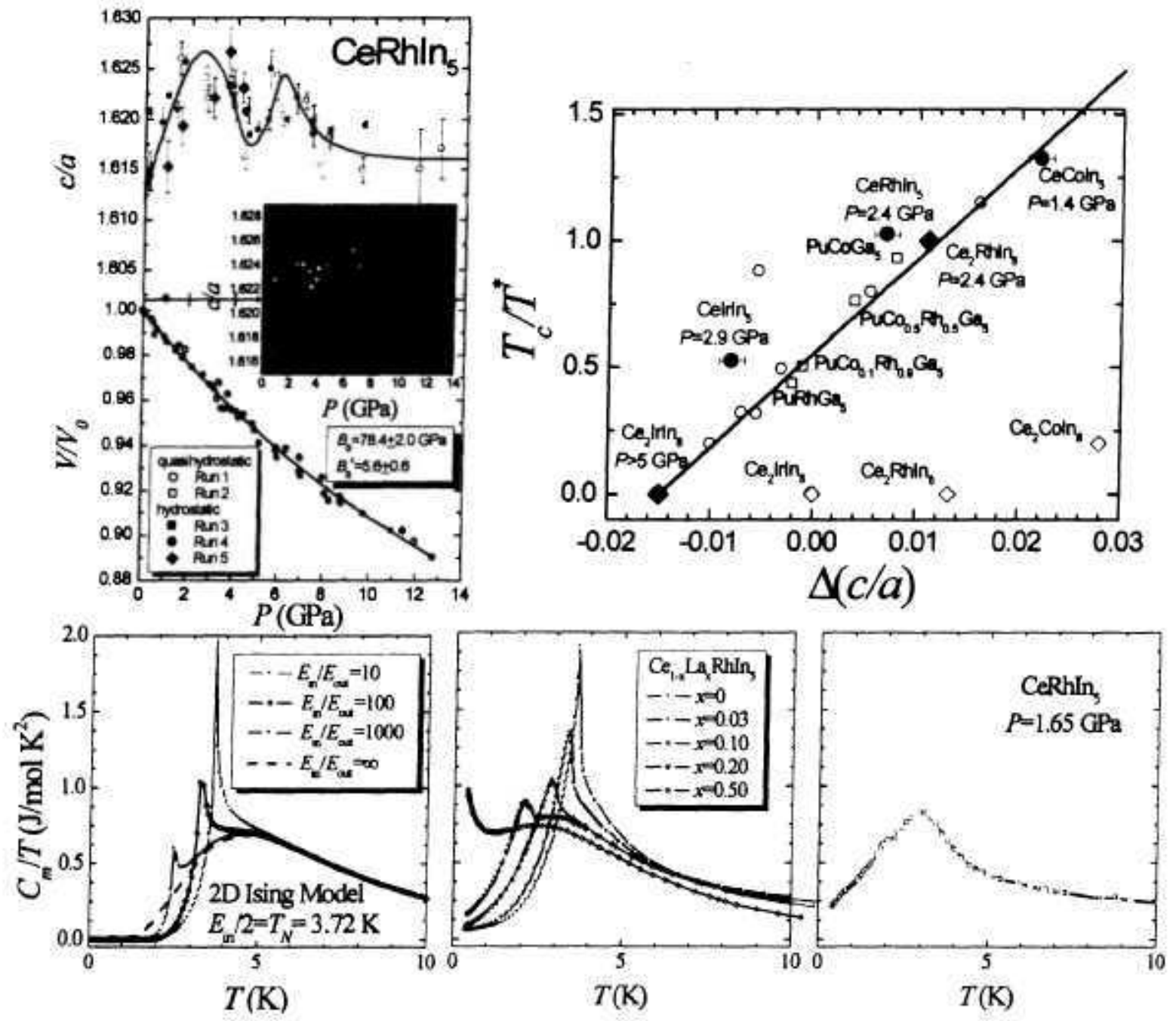

Figure 2: (Top Left) Normalized volume $V / V_{0}$ and ratio of tetragonal lattice constants $c / a$ plotted versus pressure for $\mathrm{CeRhIn}_{5}$ at room temperature. Data for both quasihydrostatic (open symbols) and hydrostatic (closed symbols) are displayed. The solid line through the volume data is a fit as described in the text. The inset shows the value of $c / a$ versus $P$ at $10 \mathrm{~K}$. (Top Right) The ambient pressure values of $T_{c} / T^{*}$ versus the room temperature value of $\Delta(c / a)$ (open symbols) for various $\mathrm{Ho}_{n} \mathrm{CoGa}_{2 n+3}$ based compounds; $\mathrm{CeM} \mathrm{In}_{5}$ (circles), $\mathrm{Ce}_{2} M \mathrm{In}_{8}$ (diamonds) and $\mathrm{Pu} M \mathrm{Ga}_{5}$ (squares) are all shown. Also shown (solid symbols) are the values of $\Delta(c / a)$ determined at room temperature at the pressure $P_{\max }$ where $T_{c}(P)$ displays a maximum. (Bottom Left) Magnetic heat capacity $C_{m}$ divided by temperature $T$ versus $T$ as calculated for the 2D Ising model described in the text. $E_{i n} / 2$ has been fixed to the value of $T_{N}=3.72 \mathrm{~K}$ for CeRhIn 5 and $E_{\text {out }}$ is varies. (Bottom Middle) $C_{m} / T$ versus $T$ measured on $\mathrm{Ce}_{1-x} \mathrm{La}_{x} \mathrm{RhIn}_{5}$ in zero field. (Bottom Right) The high pressure heat capacity data of Fischer et al. at $1.65 \mathrm{GPa}$ near the quantum critical point where a peak indicitive of short range order is seen near $3 \mathrm{~K}$ and small features due to long range order around $2.5 \mathrm{~K}$ and superconductivity near $1 \mathrm{~K}$ are also observed. ${ }^{13}$ 
alloying in a manner quite similaz to the increasing anisotropy in the 2D Ising model shown to the left. The upturn in the $\boldsymbol{x}=\mathbf{0 . 5 0}$ data is a logarithmic divergence typical of NFL behavior. The data at high pressure of Fischer $e t a \alpha^{13}$ is quite similar to the La doped anmple near the QCP. Namely, the broad maximum at $3 \mathrm{~K}$ that can be attributed to \$RO that is not shifted by magnetic feld is seen in both cases. It is natural to conclude that the NFL (upturn at low temperature in the $x=0.50$ sample) behavior in the La doped sample and the superconductivity observed under pressure in the undoped sample develop out of SRO. This behavior is consistent with an evolving picture of coexisting short and long range magnetic correlations in correlated-electron systems that holds promise for giving a universal description of these complicated materials.

\section{Publications from DOE Support 2000-2004}

- "Compressibility of $\mathrm{Ce} \mathrm{In}_{3}$ and $\mathrm{Ce}_{2} M \operatorname{In}_{8}(M=\mathrm{Rh}, \mathrm{I}$ and Co) Compounds." Ravhi S. Kumsr, A.L. Cornelius, and J.L. Sarrao, to appear in Phys. Rev. B (cond-mat/0405043)

- "X-Ray Absorption Studies of the Local Structure and f-Level Occupansy in Celr ${ }_{1-x} \mathrm{Rh}_{x} I_{n}$," M. Daniel, S.-W. Han, C.H. Booth, A.L. Cornelius, P.G. Pagliuso, J.L. Sarrao, and J.D. Thompoon, to appear in Phys. Rev. B

- "Compressibility of $\mathrm{Nb}_{2} \mathrm{AsC}$ to $41 \mathrm{GPa}$. Ravhi S. Kumar, Sandeep Rekhib, Andrew Carnelitus and Michsel W. Bersoum, submitted Appl. Phys. Lett.

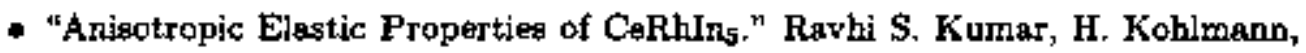
B.E. Light, A.L. Corneliu, V. Raghavan, T.W. Darling, J.L. Sarrao, P.G. Pagliuso, N.O. Moreno, and J.D. Thompson, Phys. Rev. B 69, 014515 (2004).

- "Heat Capacity Studies of Ce and Rh Site Substitution in the Heavy Fermion Antiferromagnet CeRhIn 5 : Short-Range Magnetjc Interactions and non-FermiLiquid Eehavior." B.E. Light, A.L. Cornelius, P.G. Pagliuso and J.L. Sarrao, Phys. Rev. B 69, 024419 (2004).

- "Uranium (and Cerium) Compounds At High Pressures and Magnetic Fields." A.L. Cornelius. MRS Proceedings Volume 802, DD 7.7 (2004)

" "A. New Energy Scale at Low Texnperatures in YbAlg-" T. Ebihara, A.L. Cornelius, and J.M. Lawrence, Physica B 329, 618 (2003).

- "Evolution of the Magnetic Ground State in the Electron-Doped Antiferromag-

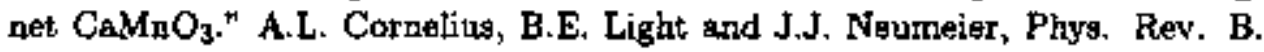
$68,014403(2003)$.

- "Dependence of the Effective Masses in $\mathrm{YbAl}_{3}$ on Magnetic Field and Disorder." T. Ebihara, E.D. Bater, A.L. Cornelius, J.M. Lawrence, N. Harribon, J.D. Thompson, J.L. Sarrao, M.F. Hundley, and S. Uji, Phys. Rev. Lett, 90, 166404 (2003).

- "Slow Crossover and Observation of a Second Energy Scale in YbAl Above 40 T." A.L. Cormelius, T. Ebihara, and J.M. Lawrence, Acta Physica Pol. B 34, 1299 (2003). 
- "Ce-Site Dilution Studies in the Aatiferromagnetic Heavy Fermions $\mathrm{Ce}_{m_{m}} \mathrm{Rh}_{\mathrm{n}} \mathrm{In}_{3 \mathrm{~m}+2 \mathrm{n}}$ $(m=1,2 ; n=0,1) . "$ P. G. Paglituso, N. O. Moreno, N. J. Curro, J. D. Thompson, M. F. Hundley, J. L. Sarrao, Z. Fisk, A. D. Christianson, A. H. Lacerda, B. E. Light, and A. L. Cornelius, Phys. Rev, B 66, 054433 (2002).

- "Observation of a Second Energy Scale in YbAl3 Above 40 T." A.L. Cornelius, T. Ebihara, J.M. Lawrence, P.S. Riseborough, and J.D. Thoupson, in Physical Phenomena in Figh Magnetic Fields IV, G. Boebinger, Z. Fisk, L. Gor'kov A. Lacerda and J.R. Schrieffer, eds., (World Scientific, 2002) 82.

- "Observation of a Second Energy Scaie in $\mathrm{YbAl}_{3}$ Above $40 \mathrm{~T}$ " A.L. Cornelius, T. Ebihara, J.M. Lawrence, P.S. Riseborough, and J.D. Thompson, Int. J. Mod. Phys. B 16, 2992 (2002).

- "Two Energy Scales and Slow Crossover in YbAil," A.L. Cornelius, J.M. Lawrence, T. Ebihara, P.S. Riseborough, C.H. Booth, M.F. Hundley, P.G. Pagliuso, J.L. Sarrao, J.D. Thompson, M.H. Jung, A.H. Lacerda, and G.H. Kwej, Phys. Rev. Lett. 88, 117201 (2002).

- "Thermodynamic lnvestigstion of the Magnetic Phase Transitions of $\mathrm{CaMnO}_{3}$ And $\mathrm{SrRuO}_{3}$." J.J. Neumejer, A.L. Cornelius, and K. Andres, Phys. Rev. B 64, 172406 (2001).

- "Field-induced Magnetic Transitions in the Quasi-Two-Dimensional Heavy Fermion Antiferromagnets $\mathrm{Ce}_{n} \mathrm{RhIn}_{3 n+2 .}$." A.L. Cornelius, P.G. Pagliuso, M.F. Hundley, and J.L. Sartao Phys. Rev. B 64, 144411 (2001).

- "Local and Average Crystal Structure and Displacements of $\mathrm{La}^{11} \mathrm{~B}_{6}$ and $\mathrm{EuB}_{6}$ as a Function of Temperature." C.H. Booth, J.L. Sarrao, M.F. Hundley, A.L.Cornelius, G.H. Kwei, A. Bianchi, Z. Fisk, and J.M. Lawrence." Phys. Rev. B 63, 224302 (2001).

- "Anisotropic Electronic and Magnetic Properties of the Quasj-Two-Dimensional Heavy-Fermion Aatiferromagnet CeRhIns." A.L. Cornelius, A.J. Arko, J.L. Sarreo, M.F. Hundley, and Z. Fisk, Phys. Rev. B 62, 14181 (2000).

\section{Invited Presentations}

- "Correlated-Electron Systems at High Pressures". A.L. Cornelius. Invited talk at the Gordon Conference Research at High Prescire, Bogton, MA. 30 minutes. June 30, 2004

- "Uranium (and Cerium) Compounds At High Pressures and Magnetic Fields." A.L. Cornelius. Invited talk at the Fatl Meeting of the Materials Resesrch Society, Boston, MA. 30 minutes. December 3, 2003.

- "Studies of Correlated-Electron Systems in High Magnetic Fitelds and ot High Pretsures." A.L. Cornelius. Invited talk at the DOE EPSCaR Conference 2003, Albuquerque, New Mexico. 20 minutes. June 4, 2003. 
- "Magnetic Measurements on $\mathrm{YOAl}_{3}$ in Pulsed Magnetic Fields to $60 \mathrm{~T}$ : The Observation of Two Energy Scales and Slow Crossover." A.L. Cornelius. Invited talk at the National High Magnetic Field Laboratory, Los Alamar, New Mexico. 60 minutes. August 23, 2002.

- "Slow crossover and observation of a second energy scale in YbAl.". A.L. Cornelius. Invited talk at the Strongly Correlated Electron Systems Conference (SCES 2002), Krakow, Poland. 20 minutes. July 10, 2002.

- "Observation of a Second Energy Scale in YbAly Above $40 \mathrm{~T}$, " Invited Talk at PPHMF-IV in Santa Fe, NM. October 21, 2001. A.L. Cornelius, T. Ebibara, J.M. Lawrence, P.S. Riseborough, and J.D. Thompson

- "Magnetic Measurements on Correlated-Elextron Systerns in Pulsed Magnetic Fields to 60 T." A.L. Cornelius, Condensed Matter Seminar at University of California, Santa Cruz. 60 minutes. February 22, 2002.

- "Suppression of Long Range Magnetic Otder." Washington University, St. Louis, April 9, 2001.

- "Studies of Magnetism and Superconductivity in Heavy Fermion Systeras." NATO Workshop Frontiers Of High Pressure Research il: Application Of High Pressure To Low-Dimensional Novel Electronic Materials, June 2001.

\section{E. Contributed Presentations}

- "Heat Capacity Studies of Ce and Rh Site Substitution in the Heavy Fermion Antiferromagnet CeRhln 5 : Short-Range Magnetic Interactions and non-FermiLiquid Behavior." B.E. Light, A.L. Corneliue, P.G. Pagliuso and J.L. Sarrao, Contributed Talk at March Meeting of the American Physical Society+ Montreal, QUE, 15 minutes, March 22, 2004.

- "X-Ray Absorption Studieg of the Local Structure and Electronic Configurstion in Celr ${ }_{1-z} \mathrm{Rh}_{z} \mathrm{Ins}_{\text {s." }}$ M. Daniei, C.H. Boath, A.L. Cornelius, S.-W. Han and J.L. Sarrao, Contributed Talk at March Meeting of the American Physical Society, Montreal, QUE, 15 minutes, March 22, 2004.

- "Spin Relaxation Processes in the Frustrated Ising System Hos Ru $\mathrm{O}_{7}$." A.L. Cornelius and 1.S. Gardner, Contributed Talk at March Meeting of the American Physical Society, Montreal, QUE, 15 minutes, March 22, 2004.

- "Structural Studies of Lar-2x $\mathrm{Sr}_{1+2 x} \mathrm{Mr}_{2} \mathrm{O}_{7}$ Bilayer Manganites." Ravhi Kumar, YongRong Shen, Andrew. Cornelius, Prabhakaraj Dharmalingam, and Andrew Boothroyd, Contributed Talk at March Meeting of the American Physical Society, Montreal, QUE, 15 minutes, March 23, 2004.

- "Preseure jnduced amorphization of $\mathrm{Tb}_{2} \mathrm{Ti}_{2} \mathrm{O}_{7}$ pyrochlore." Ravhi Kumar, YongRong Shen, Andrew. Cornelius, and Jason Gardner, Contributed Talk at March Meeting of the American Physical Society, Montres, QUE, 15 minutes, March 23, 2004. 
- "Low Temperature, High Pressure Physics," A.L. Cornelius. Contributed pre sentation at UNLV-DOE Nevada Stockpile Stewardship Review. 30 minutes. Decennber 5, 2003.

- "A New Intermediate Amorphous Phase Pathway in B1 B2 Transition." Ravhi S. Kumar, Yongrong Shen, Eunja Kim, Andrew Cornelius, Changteng Chen, Malcolm F Nicol, and S. Yoneda. Contributed paster. Gordon Conference Research at High Pressure, Boston, MA. 30 minutes. June 28, 2004.

- "Anisotropic Elastjc Properties of CeRhIns." Ravhi S. Kumar, H. Kohlmann, B.E. Light, A.L. Cornelius, V. Raghavan, T.W. Darling, J.L. Sartao, P.G. Peglínso, N.O. Moreno, and J.D. Thornpson, Contributed Talk at March Meeting of the American Physical Society, Austin, TX, 15 minutes, March 6, 2003.

- "Evolution of the Magnetic Ground State in the Electron-Doped Antiferromagnet $\mathrm{CaMnO}_{3} .^{n}$ B.E. Light, A.L. Cornelius, and J.J. Neurneier, Contributed Talk at March Meeting of the American Physical Society, Austin, TX, 15 minutes, March 52003.

"Field Dependent Effective Marsee in YbAl3." T. Ebihara, A.L. Cornelius, J.M. Lawrence, S. Uji, and N. Harrigon, Contributed Talk at March Meting of the American Pbysical Society, Austin, TX, 15 minutes, March 4, 2003.

- "Obervation of Multiple Spin Relaxation Processes in Dysprogium Titanate." A.L. Cornelius, B. Yuiga, B. Light, and J. Gardner, Contributed Talk at March Meeting of the American Physical Society, Austin, TX, 15 minutes, March 4, 2003 .

- "Studies of Magnetism in Correlated-Electron Syatems." A.L. Corneliws. Contributed presentation at UNLV-DOE Nevada Stockpile Stewardship Review. 30 minutes. November 2, 2002 .

- "A new energy scale at low temperature in YbAlg." T. Ebihara, A.L. Cornelius, and J.M. Lawtence. Contributed talk. 23rd Int. Conf. on Low Temperature Physics, Hiroshima, Japan, August 23, 2002.

- "Studies of Correlated-Electron Systems in High Magnetic Fields and at High Pressures." A.L. Cornelius. Contributed prentation DOE/EPSCoR Site Visit for the University/National Laboratory Collaboration Program. 15 minutes. February 26, 2002.

- "Anisotropic Elastic Properties of CeRhIn $n_{5}$ and Related Compounds." Ravhi S. Kumar, H. Kohlmann, B.E. Light, A.L. Cornelius, V. Raghavan, T.W. Darling, J.L. Sarrao, P.G. Pagliuso, N.O. Moreno, and J.D. Thompeon. Pogter presented at the Gordon Conference entitled Rosearch at Figh Pressure. June 25, 2002.

- "Studieg of Magnetism in Correlated-Electron Systems." A.L. Cornelius. Contributed presentation at the Annual Review of the DOE Nevada Stockpile Stewardship Program. 15 minutes. September 23, 2001. 
- "de Hass-van Alphen effect, magnetic transitions and specific heat in the heavy fermion antiferromagnet CeRhIns." A.L. Cornelius, A.J. Arko, 3.L. Sarrao, M.F. Hundkey, J.D. Thompson, and N. Harrison, Contributed Talk at March Meeting of the American Physical Society, Minneapolis, MN, 15 minutes, March 23, 2001. 


\section{Research Bibliography}

IA L Cornelius, J M Lawrence, T Ebihara, P S Risebrorough, C H Booth, M F Hundley, P G Pagliuso, J L Sarrao, J D Thompson, M H Jung, A H Lacerda, and G H Kwei, "Two energy scales and slow crossover in YbAl 3 ", Phys. Reu. Lett., vol. 88, pp. 117201, 2002.

${ }^{2}$ A N Tahvildar-Zaden, M Jarrell, and J K Freericks, "Low-temperature coherence in the periodic Anderson model: Predictions for photoenission of heavy fermions", Phys. Rev. Lett., vol. 80, pp. \$168, 1998.

'S Burdin, A Georges, and D R Grempel, "Coherence scale of the Kondo lattice", Phys. Rev. Lett. vol. 85, pp. $1048,2000$.

"F F Assaad, "Coberence scale of the two-dimensional Kondo lattice model", Phys. Rev. $B_{1}$ vol. 70, pp. 020402R, 2004.

ST Ebihara, E D Bauer, A L Cornelius, J M Lawrence, N Harrison, J D Thompson, $J \mathcal{L}$ Sarrao, M F Hundley, and $S \mathrm{Ujt}^{\mathrm{j}}$, "Dependence of the effective masses in $\mathrm{YbAl}_{3}$ on magnetic field and disorder ${ }^{n}$, Phys. Rev. Lett., vol. 90, pp. 166404, 2003 .

${ }^{6}$ H Hegger, C Petrovic, E G Moshopoulou, M F Hundley, J L Sarrao, Z Fisk, and J D Thompson, "Pressure-induced superconductivity in quasi-2d CeRhing", Phys. Rev. Lett., vol. 84, pp. 4986, 2000.

${ }^{7}$ A L Cornelim, A J Arko, J L Sarrao, M F Hundley, and Z Fisk, "Anisotropic electronic and magnetic properties of the quasi-two-dimensional hesvy-fermion antiferrornagnet CeRhins", Phys. Rev. B, vol. 62, PP. 14181, 2000.

${ }^{8} \mathrm{P}$ Monthoux and G G Lonzarich, "Magnetically mediated superconductivity in quasj-two and three dimensions", Phys. Rev. B, vol. 63, pp. 054529, 2001.

${ }^{9}$ W Bao, P G Pagliuso, J L Sarrao, J D Thompson, Z Fisk, J W Lynn, and R W Erwin, "Incommensurate magnetic atructure of CeRhlns", Phys. Ret. B, vol. 62, pp. R14621, 2000.

${ }^{10}$ I Vedel, A M Redon, J M Mignot, and J M Leger, "Absence of discontinuity in the pressure-votume dependence of $\mathrm{CeAl}_{2}$ and $\mathrm{CeIn}_{3}{ }^{n}, J$. Phys. F: Metal Phys, vol. 17, pp. 849, 1987.

${ }^{11}$ T Muramatsu, N Tateiwa, T C Kobayashi, AK Shimizu, K Amays, D Aoki, H Shishido, Y Haga, and Y Onukj, "Superconductivity of CeRhIn, under high pressure", J. Phys. Soc. Jpn., vol. 70, pp. 3362, 2001.

${ }^{12}$ M Nicklas, V A Sidorov, H A Borges, P G Paglinso, J L Sarrao, and J D Thompson, "Two superconducting phases in CeRh ${ }_{1-x} \mathrm{Ir}_{\mathrm{x}} \mathrm{In}_{\mathrm{s}}$ ", Phys. Rev. B, vol. 70, pp. $020505(\mathrm{R}), 2004$. 
${ }^{13}$ R A Fiaher, F Bouquet, N E Phillips, M F Hundley, P G Pagliuso, J L Sarrao, Z Fisk, and J D Thompeon, "Specific heat of CeRhing: Pressure-driven evolution of the ground state from antiferromagnetisa to superconductjvity", Phys. Rev. B, vol. 65, pp. 224509, 2092.

${ }^{14} \mathrm{P}$ G Pagliuso, A Morshovich, A D Bianchi, M Nicklas, N O Moreno, I D Thompson, M F Hundley, J L Sarrno, and Z Fisk, "Multiple phase transitions in Ce(Rh,Ir,Co)Ins", Physica B, vol. 3I2-313, pp. 129, 2002.

${ }^{15}$ S Nakatsuji, S Yeo, L Balicas, Z Fisk, P Schlottmann, P G Pagliuso, N O Moreno, J L Sarrao, and J D Thompson, "Intersite coupling effects in a kondo lattíce", Phys. Rey. Lett. vol. 89, pp. 106402, 2002.

${ }^{16}$ F Wastin, P Boulet, J Rebizant, E Colineau, and G H Lander, "Advances in the preparation and characterization of transuranium systems", J. Phys.:Condent. Motter, vol. 15, pp. S2279, 2003.

${ }^{17}$ F Wastin, P Boulet, E Colineau, I Rebizant, G H Lander, J D Thoupson, J L Sarrao, and L A Morales, "Superconductivity in plutopium based compounds", 2004.

${ }^{18} \mathrm{~B} M$ McCoy and T T Wu, Two Two-Dimensional Ssing Model, Harvard University Press, Cambridge, 1973. 